\title{
Stochastic sensitivity analysis using HDMR and score function
}

\author{
RAJIB CHOWDHURY ${ }^{1}$, B N RAO $^{* 2}$ and A MEHER PRASAD ${ }^{2}$ \\ ${ }^{1}$ School of Engineering, Swansea University, Swansea, SA2 8PP, UK \\ ${ }^{2}$ Structural Engineering Division, Department of Civil Engineering, Indian \\ Institute of Technology Madras, Chennai 600036 \\ e-mail: bnrao@iitm.ac.in
}

MS received 14 November 2008; revised 30 August 2009

\begin{abstract}
Probabilistic sensitivities provide an important insight in reliability analysis and often crucial towards understanding the physical behaviour underlying failure and modifying the design to mitigate and manage risk. This article presents a new computational approach for calculating stochastic sensitivities of mechanical systems with respect to distribution parameters of random variables. The method involves high dimensional model representation and score functions associated with probability distribution of a random input. The proposed approach facilitates firstand second-order approximation of stochastic sensitivity measures and statistical simulation. The formulation is general such that any simulation method can be used for the computation such as Monte Carlo, importance sampling, Latin hypercube, etc. Both the probabilistic response and its sensitivities can be estimated from a single probabilistic analysis, without requiring gradients of performance function. Numerical results indicate that the proposed method provides accurate and computationally efficient estimates of sensitivities of statistical moments or reliability of structural system.
\end{abstract}

Keywords. Stochastic sensitivity; structural reliability; high dimensional model representation; score function; statistical moment.

\section{Introduction}

Sensitivity analysis provides an important insight towards understanding the physical mechanisms underlying failure and modifying the design towards mitigating risk. Significant advancements have been made over the past few decades in developing methods such that the sensitivity information is provided as a by-product of the analysis or at a significantly reduced cost with improved accuracy. Studies on sensitivities of the random variables are carried out in the literature (Melchers \& Ahammed 2004; Au 2005) in order to establish a systematic framework for identifying relative importance of parameters that merit

*For correspondence 
descriptions through random variables or optimizing a system's performance with an acceptable risk. For estimating the sensitivity of a general probabilistic response, there are three broad approaches, namely, finite-difference method (L'Ecuyer \& Perron 1994), perturbation analysis (Ho \& Cao 1991) and score function/likelihood method (Rubinstein \& Shapiro 1993). The finite-difference method involves repeated stochastic analyses for nominal and perturbed values of system parameters, and then invoking differentiation schemes to approximate their partial derivatives. This method is often expensive because evaluating probabilistic response for each system parameter, which constitutes a complete stochastic analysis, is a computationally challenging task. Remaining methods are mostly viewed as competing methods, where both performance and sensitivities can be obtained from a single stochastic simulation. For perturbation analysis (Glasserman 1991), the probability measure is fixed, and the gradient of a performance function is taken, assuming that the differential and integral operators are interchangeable. Score function method (Rubinstein \& Shapiro 1993), which involves probability measure that continuously varies with respect to a design parameter, also requires a somewhat similar interchange of differentiation and integration, but in many practical examples, interchange in the score function method holds in a much wider range than that in perturbation analysis. However, both methods are typically employed in conjunction with the direct Monte Carlo simulation (MCS), a premise well-suited to stochastic optimization of discrete event systems. Unfortunately, gradient estimation of a stochastic system, where stochastic response and sensitivity analyses are required at each realization, even a single cycle of MCS is impractical, as each deterministic trial of the simulation may require expensive finite element (FE) or other numerical calculations. This is the principal reason why neither the perturbation analysis nor the score function method have found their way in to the stochastic optimization of mechanical systems.

The direct differentiation method, commonly used in deterministic sensitivity analysis (Haug et al 1986), provides an attractive alternative to the finite-difference method for calculating stochastic sensitivities. In conjunction with first-order reliability method (FORM), Liu \& Der Kiureghian (1991) have significantly contributed to the development of such methods for obtaining reliability sensitivities. The direct differentiation method, also capable of generating both reliability and its sensitivities from a single stochastic analysis, is particularly effective in solving FE-based reliability problems, when (a) the most probable point can be efficiently located and (b) a linear approximation of the performance function at that point is adequate. In contrast, the three sensitivity methods described in the preceding are independent of underlying stochastic analysis. In this article, high dimensional model representation (HDMR) is used in conjunction with score function for calculating stochastic sensitivities of structural/mechanical system with respect to probability distribution parameters.

The paper is organized as follows. Section 2 presents a brief overview of statistical moments. Section 3 describes the concept of sensitivity analysis using score function. Section 4 presents a brief overview of HDMR and its applicability to reliability analysis. Section 5 presents approximation of the original implicit limit state/performance function using HDMR. Section 6 presents the estimation of failure probability, statistical moments and sensitivities by MCS using the approximate limit state/performance function generated by HDMR. Numerical examples involving elementary mathematical functions and structural problems are presented in Section 7 to illustrate the proposed method. 


\section{Statistical moments}

Let $\boldsymbol{x}=\left\{x_{1}, x_{2}, \ldots, x_{N}\right\}$ denote a family of $\mathfrak{R}^{N}$-valued input random vector, describing uncertainties in loads, material properties and geometry of structural/mechanical system. The probability law of the random variables is completely defined by joint density function $\left\{f_{\boldsymbol{x}}(\boldsymbol{x} ; \boldsymbol{\theta}), \boldsymbol{x} \in \mathfrak{R}^{N}, \boldsymbol{\theta} \in \mathfrak{R}^{N}\right\}$ that is associated with probability measure $\left\{P_{\boldsymbol{\theta}}, \boldsymbol{\theta} \in \mathfrak{R}^{N}\right\}$. It is assumed that the performance/limit state function $g(\boldsymbol{x})$ is not an explicit function of $\boldsymbol{\theta}$, although $g(\boldsymbol{x})$ implicitly depends on $\boldsymbol{\theta}$ through the probability law of $\boldsymbol{x}=\left\{x_{1}, x_{2}, \ldots, x_{N}\right\}$. The objective of probabilistic sensitivity analysis is to obtain the partial derivatives of a probabilistic characteristic of $g(\boldsymbol{x})$ with respect to a parameter $\theta_{i}, i=1,2, \ldots, M$, given a reasonably arbitrary probability law of $\boldsymbol{x}=\left\{x_{1}, x_{2}, \ldots, x_{N}\right\}$.

The $q^{\text {th }}$ moment of $g(\boldsymbol{x})$ can be defined by

$$
m_{q}(\boldsymbol{\theta})=E_{\boldsymbol{\theta}}\left[g^{q}(\boldsymbol{x})\right]=\int_{\mathfrak{R}^{N}} g^{q}(\boldsymbol{x}) f_{X}(\boldsymbol{x} ; \boldsymbol{\theta}) d \boldsymbol{x} ; \quad q=1,2, \ldots
$$

A similar integral appears in time-invariant reliability analysis, which entails calculating the failure probability

$$
P_{F}(\boldsymbol{\theta})=P_{\boldsymbol{\theta}}\left[\boldsymbol{x} \in \Omega_{F}\right]=\int_{\Re^{N}} \mathcal{J}_{\Omega_{F}}(\boldsymbol{x}) f_{X}(\boldsymbol{x} ; \boldsymbol{\theta}) d \boldsymbol{x},
$$

where $\Omega_{F}$ is the failure set for component reliability analysis and

$$
\mathcal{J}_{\Omega_{F}}(\boldsymbol{x})=\left\{\begin{array}{ll}
1, & x \in \Omega_{F} \\
0, & x \in \Omega \backslash \Omega_{F}
\end{array} ; \quad x \in \Re^{N},\right.
$$

is an indicator function. Therefore, both the expressions in (2) and (3) can be consolidated into a generic probabilistic response

$$
h(\boldsymbol{\theta})=E_{\boldsymbol{\theta}}[g(\boldsymbol{x})]=\int_{\Re^{N}} g(\boldsymbol{x}) f_{X}(\boldsymbol{x} ; \boldsymbol{\theta}) d \boldsymbol{x} .
$$

\section{Sensitivity analysis using score function}

The score functions depend only on the probability distribution of random input $\boldsymbol{x}=$ $\left\{x_{1}, x_{2}, \ldots, x_{N}\right\}$. When the distribution of $x_{i}$ is either independent or dependent, the expressions of the score functions simplify slightly. Since a major application of sensitivity analysis is design optimization, where the second-moment properties of random input play the role of design parameters, attention is confined to the score functions associated with the mean and standard deviations of input.

\subsection{Independent distribution}

Consider a distribution parameter $\theta_{i}, i=1,2, \ldots, M$, and suppose that the gradient of a generic probabilistic response $h(\boldsymbol{\theta})$, which is either statistical moment or reliability of a structural/mechanical system, with respect to $\theta_{i}$ is sought. According to Rubinstein \& Shapiro (1993), pioneer of the score function method, few assumptions are necessary for 
such sensitivity analysis. Details of these assumptions can be found in Rubinstein \& Shapiro (1993). Taking the partial derivative of both sides of (4) with respect to $\theta_{i}$ yields

$$
\frac{\partial h(\boldsymbol{\theta})}{\partial \theta_{i}}=\frac{\partial}{\partial \theta_{i}} \int_{\Re^{N}} g(\boldsymbol{x}) f_{X}(\boldsymbol{x} ; \boldsymbol{\theta}) d x .
$$

By invoking Lebesgue dominated convergence theorem, the differential and integral operators can be interchanged, which yields

$$
\begin{aligned}
\frac{\partial h(\boldsymbol{\theta})}{\partial \theta_{i}} & =\int_{\Re^{N}} g(\boldsymbol{x}) \frac{\partial f_{X}(\boldsymbol{x} ; \boldsymbol{\theta})}{\partial \theta_{i}} d \boldsymbol{x}=\int_{\Re^{N}} g(\boldsymbol{x}) \frac{\partial \ln f_{X}(\boldsymbol{x} ; \boldsymbol{\theta})}{\partial \theta_{i}} f_{X}(\boldsymbol{x} ; \boldsymbol{\theta}) d \boldsymbol{x} \\
& =E_{\theta}\left[g(\boldsymbol{x}) \frac{\partial \ln f_{X}(\boldsymbol{x} ; \boldsymbol{\theta})}{\partial \theta_{i}}\right] ; \quad i=1, \ldots, M .
\end{aligned}
$$

Define

$$
K_{\theta}^{(1)}(\boldsymbol{x} ; \boldsymbol{\theta})=\frac{\partial \ln f_{\boldsymbol{x}}(\boldsymbol{x} ; \boldsymbol{\theta})}{\partial \theta_{i}},
$$

which is known as the first-order score function for the parameter $\theta_{i}$.

\subsection{Dependent distribution}

When the probability distribution of random input $\boldsymbol{x}=\left\{x_{1}, x_{2}, \ldots, x_{N}\right\}$ is dependent, the derivation of score functions is generally tedious. For example, when $\boldsymbol{x}=\left\{x_{1}, x_{2}, \ldots, x_{N}\right\}$ is following Gaussian distribution with mean $\boldsymbol{\mu}=\left\{\mu_{1}, \mu_{2}, \ldots, \mu_{N}\right\}$ and covariance $\Sigma=$ $E_{\boldsymbol{\theta}}\left[(\boldsymbol{x}-\boldsymbol{\mu})(\boldsymbol{x}-\boldsymbol{\mu})^{T}\right]=\left[\rho_{i j} \sigma_{i} \sigma_{j}\right]$ and joint density $f_{\boldsymbol{x}}(\boldsymbol{x} ; \boldsymbol{\theta})=\left[(2 \pi)^{N / 2}|\Sigma|^{0 \cdot 5}\right]^{-1} \exp [-(\boldsymbol{x}-$ $\boldsymbol{\mu}) \Sigma^{-1}(\boldsymbol{x}-\boldsymbol{\mu})^{T}$, where $\mu_{i}$ and $\sigma_{i}$ are the mean and standard deviation, respectively, of $x_{i}$ and $\rho_{i j}$ is the correlation coefficient between $x_{i}$ and $x_{j}$, the first-order score functions are

$$
K_{\mu_{i}}^{(1)}(\boldsymbol{x} ; \boldsymbol{\theta})=\{0, \ldots, 1, \ldots, 0\} \Sigma^{-1}(\boldsymbol{x}-\boldsymbol{\theta}),
$$

and

$$
K_{\sigma_{i}}^{(1)}(\boldsymbol{x} ; \boldsymbol{\theta})=-\frac{1}{2}(\boldsymbol{x}-\boldsymbol{\theta})^{T} \frac{\partial \Sigma^{-1}}{\partial \sigma_{i}}(\boldsymbol{x}-\boldsymbol{\theta})-\frac{1}{2} \frac{\partial \ln |\Sigma|}{\partial \sigma_{i}} .
$$

Therefore, the first-order sensitivity of $h(\theta)$ can be expressed by

$$
\frac{\partial h(\boldsymbol{\theta})}{\partial \theta_{i}}=E_{\theta}\left[g(\boldsymbol{x}) K_{\theta}^{(1)}(\boldsymbol{x} ; \boldsymbol{\theta})\right] ; \quad i=1, \ldots, M .
$$

The score function method requires differentiating only the probability density function. Also in most cases, resulting score functions can be easily determined analytically. In contrast, the perturbation analysis requires derivatives or perturbation of the limit state/performance function, which is always expensive in stochastic mechanics applications. Furthermore, if the performance function is not differentiable, interchangeability of differential and integral operators is violated and the direct differentiation based approaches will not work. In the score function method, $g(\boldsymbol{x})$ can be discontinuous - for example, the indicator function $\mathcal{J}_{\Omega_{F}}(\boldsymbol{x})$ that comes from reliability analysis - but the method still allows evaluation of the sensitivity if the density function is differentiable. Due to these facts the score function method is chosen in this paper, as a tool for efficient computation of probabilistic sensitivity. 


\section{High dimensional model representation}

The fundamental principle underlying the HDMR (Rabitz \& Alis 1999; Rabitz et al 1999; Wang et al 1999; Alis \& Rabitz, 2001; Li et al 2001a; Li et al 2001b; Sobol, 2003) is that, from the perspective of the output/response, the order of cooperative effects between the independent variables will die off rapidly. This assertion does not eliminate strong variable dependence or even the possibility that all the variables are important. Various sources (Rabitz \& Alis 1999; Wang et al 1999; Alis \& Rabitz 2001) of information support this point of there being limited high-order correlations. First, the variables in most systems are chosen to enter as independent entities. Second, traditional statistical analyses of system behavior have revealed that a variance and covariance analysis of the output in relation to the input variables often adequately describes the physics of the problem. These general observations lead to a dramatically reduced computational scaling when one seeks to map input-output relationships of complex systems.

Evaluating the input-output mapping of the system generates a HDMR. This is achieved by expressing system response as a hierarchical, correlated function expansion of a mathematical structure and evaluating each term of the expansion independently. One may show that system response that is a function of $N$ input variables, $g(\boldsymbol{x})=g\left(x_{1}, x_{2}, \ldots, x_{N}\right)$, can be expressed as summands of different dimensions:

$$
\begin{aligned}
g(\boldsymbol{x})= & g_{0}+\sum_{i=1}^{N} g_{i}\left(x_{i}\right)+\sum_{1 \leq i_{1}<i_{2} \leq N} g_{i_{1} i_{2}}\left(x_{i_{1}}, x_{i_{2}}\right)+\cdots \\
& +\sum_{1 \leq i_{1}<\cdots<i_{l} \leq N} g_{i_{1} i_{2} \ldots i_{l}}\left(x_{i_{1}}, x_{i_{2}}, \ldots, x_{i_{l}}\right)+\cdots+g_{12 \ldots N}\left(x_{1}, x_{2}, \ldots, x_{N}\right),
\end{aligned}
$$

where $g_{0}$ is a constant term representing the mean response of $g(\boldsymbol{x})$. The function $g_{i}\left(x_{i}\right)$ describes the independent effect of variable $x_{i}$ acting alone, although generally nonlinearly, upon the output $g(\boldsymbol{x})$. The function $g_{i_{1} i_{2}}\left(x_{i_{1}}, x_{i_{2}}\right)$ gives pair correlated effect of the variables $x_{i_{1}}$ and $x_{i_{2}}$ upon the output $g(\boldsymbol{x})$. The last term $g_{12 \ldots N}\left(x_{1}, x_{2}, \ldots, x_{N}\right)$ contains any residual correlated behaviour over all of the system variables. Usually the higher order terms in (11) are negligible (Rabitz \& Alis 1999; Rabitz et al 1999) such that HDMR with only low order correlations to second-order (Li et al 2001a), amongst the input variables are typically adequate in describing the output behaviour.

The expansion functions are determined by evaluating the input-output responses of the system relative to the defined reference point $c=\left\{c_{1}, c_{2}, \ldots, c_{N}\right\}$ along associated lines, surfaces, subvolumes, etc. (i.e. cuts) in the input variable space. This process reduces to the following relationship for the component functions in (11)

$$
\begin{aligned}
& g_{0}=g(\boldsymbol{c}), \\
& g_{i}\left(x_{i}\right)=g\left(x_{i}, \boldsymbol{c}^{i}\right)-g_{0}, \\
& g_{i_{1} i_{2}}\left(x_{i_{1}}, x_{i_{2}}\right)=g_{i_{1} i_{2}}\left(x_{i_{1}}, x_{i_{2}}, \boldsymbol{c}^{i_{1} i_{2}}\right)-g_{i_{1}}\left(x_{i_{1}}\right)-g_{i_{2}}\left(x_{i_{2}}\right)-g_{0},
\end{aligned}
$$

where the notation $g\left(x_{i}, \boldsymbol{c}^{i}\right)=g\left(c_{1}, c_{2}, \ldots, c_{i-1}, x_{i}, c_{i+1}, \ldots, c_{N}\right)$ denotes that all the input variables are at their reference point values except $x_{i}$. The $g_{0}$ term is the output response of the system evaluated at the reference point $c$. The higher order terms are evaluated as 
cuts in the input variable space through the reference point. Therefore, each first-order term $g_{i}\left(x_{i}\right)$ is evaluated along its variable axis through the reference point. Each second-order term $g_{i_{1} i_{2}}\left(x_{i_{1}}, x_{i_{2}}\right)$ is evaluated in a plane defined by the binary set of input variables $x_{i_{1}}, x_{i_{2}}$ through the reference point, etc. The process of subtracting off the lower order expansion functions removes their dependence to assure a unique contribution from the new expansion function.

Considering terms up to first- and second-order in (11) yields first- and second-order HDMR approximation of $g(\boldsymbol{x})$ as

$$
\tilde{g}(\boldsymbol{x})=\sum_{i=1}^{N} g\left(c_{1}, \ldots, c_{i-1}, x_{i}, c_{i+1}, \ldots, c_{N}\right)-(N-1) g(\boldsymbol{c}),
$$

and

$$
\begin{aligned}
\tilde{g}(\boldsymbol{x})= & \sum_{\substack{i_{1}=1, i_{2}=1 \\
i_{1}<i_{2}}}^{N} g\left(c_{1}, \ldots, c_{i_{1}-1}, x_{i_{1}}, c_{i_{1}+1}, \ldots, c_{i_{2}-1}, x_{i_{2}}, c_{i_{2}+1}, \ldots, c_{N}\right) \\
& -(N-2) \sum_{i=1}^{N} g\left(c_{1}, \ldots, c_{i-1}, x_{i}, c_{i+1}, \ldots, c_{N}\right)+\frac{(N-1)(N-2)}{2} g(\boldsymbol{c})
\end{aligned}
$$

respectively. It can also be noted that, compared with FORM and SORM which retains only linear and quadratic terms, respectively, first-order HDMR provides more accurate approximation $\tilde{g}(\boldsymbol{x})$ of the original implicit limit state/performance function $g(\boldsymbol{x})$ (Chowdhury et al 2009). If first-order HDMR approximation is not sufficient the second-order HDMR approximation may be adopted at the expense of additional computational cost.

\section{Generation of HDMR approximation}

HDMR in (11) is exact along any of the cuts and the output response $g(\boldsymbol{x})$ at a point $\boldsymbol{x}$ off of the cuts can be obtained by following the procedure in step 1 and step 2 below:

Step 1: Interpolate each of the low dimensional HDMR expansion terms with respect to the input values of the point $\boldsymbol{x}$. For example, consider the first-order component function $g\left(x_{i}, \boldsymbol{c}^{i}\right)=g\left(c_{1}, c_{2}, \ldots, c_{i-1}, x_{i}, c_{i+1}, \ldots, c_{N}\right)$. If for $x_{i}=x_{i}^{j}, n$ function values

$$
g\left(x_{i}^{j}, c^{i}\right)=g\left(c_{1}, \ldots, c_{i-1}, x_{i}^{j}, c_{i+1}, \ldots, c_{N}\right) ; \quad j=1,2, \ldots, n,
$$

are given at $n(=3,5,7$ or 9$)$ equally spaced sample points $\mu_{i}-(n-1) \sigma_{i} / 2, \mu_{i}-(n-$ 3) $\sigma_{i} / 2, \ldots, \mu_{i}, \ldots, \mu_{i}+(n-3) \sigma_{i} / 2, \mu_{i}+(n-1) \sigma_{i} / 2$ along the variable axis $x_{i}$ with mean $\mu_{i}$ and standard deviation $\sigma_{i}$, the function value for arbitrary $x_{i}$ can be obtained by the moving least square (MLS) interpolation (Lancaster \& Salkauskas, 1986) as

$$
g\left(x_{i}, \boldsymbol{c}^{i}\right)=\sum_{j=1}^{n} \phi_{j}\left(x_{i}\right) g^{\prime}\left(c_{1}, \ldots, c_{i-1}, x_{i}^{j}, c_{i+1}, \ldots, c_{N}\right),
$$


where

$$
\left\{\begin{array}{c}
g^{\prime}\left(x_{i}^{1}, \boldsymbol{c}^{i}\right) \\
\vdots \\
\vdots \\
g^{\prime}\left(x_{i}^{n}, \boldsymbol{c}^{i}\right)
\end{array}\right\}=\left[\begin{array}{cccc}
\phi_{1}\left(x_{i}^{1}\right) & \phi_{2}\left(x_{i}^{1}\right) & \cdots & \phi_{n}\left(x_{i}^{1}\right) \\
\vdots & \vdots & \vdots & \vdots \\
\vdots & \vdots & \vdots & \vdots \\
\phi_{1}\left(x_{i}^{n}\right) & \phi_{2}\left(x_{i}^{n}\right) & \cdots & \phi_{n}\left(x_{i}^{n}\right)
\end{array}\right]^{-1}\left\{\begin{array}{c}
g\left(x_{i}^{1}, \boldsymbol{c}^{i}\right) \\
\vdots \\
\vdots \\
g\left(x_{i}^{n}, \boldsymbol{c}^{i}\right)
\end{array}\right\}
$$

Similarly, consider the second-order component function $g\left(x_{i_{1}}, x_{i_{2}}, c^{i_{1} i_{2}}\right)=g\left(c_{1}, \ldots, c_{i_{1}-1}\right.$, $\left.x_{i_{1}}, c_{i_{1}+1}, \ldots, c_{i_{2}-1}, x_{i_{2}}, c_{i_{2}+1}, \ldots, c_{N}\right)$. If for $x_{i_{1}}=x_{i_{1}}^{j_{1}}$, and $x_{i_{2}}=x_{i_{2}}^{j_{2}}, n^{2}$ function values

$$
\begin{aligned}
& g\left(x_{i_{1}}^{j_{1}}, x_{i_{2}}^{j_{2}}, c^{i_{1} i_{2}}\right)=g\left(c_{1}, \ldots, c_{i_{1}-1}, x_{i_{1}}^{j_{1}}, c_{i_{1}+1}, \ldots, c_{i_{2}-1}, x_{i_{2}}^{j_{2}}, c_{i_{2}+1}, \ldots, c_{N}\right) \\
& \quad j_{1}=1,2, \ldots, n, \quad j_{2}=1,2, \ldots, n
\end{aligned}
$$

are given on a grid formed by taking $n\left(=3,5,7\right.$ or 9) equally spaced sample points $\mu_{i_{1}}-$ $(n-1) \sigma_{i_{1}} / 2, \mu_{i_{1}}-(n-3) \sigma_{i_{1}} / 2, \ldots, \mu_{i_{1}}, \ldots, \mu_{i_{1}}+(n-3) \sigma_{i_{1}} / 2, \mu_{i_{1}}+(n-1) \sigma_{i_{1}} / 2$ along $x_{i_{1}}$ axis with mean $\mu_{i_{1}}$ and standard deviation $\sigma_{i_{1}}$, and $n(=3,5,7$ or 9$)$ equally spaced sample points $\mu_{i_{2}}-(n-1) \sigma_{i_{2}} / 2, \mu_{i_{2}}-(n-3) \sigma_{i_{2}} / 2, \ldots, \mu_{i_{2}}, \ldots, \mu_{i_{2}}+(n-3) \sigma_{i_{2}} / 2, \mu_{i_{2}}+(n-1) \sigma_{i_{2}} / 2$ along $x_{i_{2}}$ axis with mean $\mu_{i_{2}}$ and standard deviation $\sigma_{i_{2}}$, the function value for arbitrary $\left(x_{i_{1}}, x_{i_{2}}\right)$ can be obtained by the MLS interpolation as

$$
\begin{aligned}
g\left(x_{i_{1}}, x_{i_{2}}, \boldsymbol{c}^{i_{1} i_{2}}\right)= & \sum_{j_{1}=1}^{n} \sum_{j_{2}=1}^{n} \phi_{j_{1} j_{2}}\left(x_{i_{1}}, x_{i_{2}}\right) \\
& \times g^{\prime}\left(c_{1}, \ldots, c_{i_{1}-1}, x_{i_{1}}^{j_{1}}, c_{i_{1}+1}, \ldots, c_{i_{2}-1}, x_{i_{2}}^{j_{2}}, c_{i_{2}+1}, \ldots, c_{N}\right),
\end{aligned}
$$

where

$$
\begin{gathered}
\left\{\begin{array}{c}
g^{\prime}\left(x_{i_{1}}^{1}, x_{i_{2}}^{1}, \boldsymbol{c}^{i_{1} i_{2}}\right) \\
\vdots \\
g^{\prime}\left(x_{i_{1}}^{1}, x_{i_{2}}^{n}, \boldsymbol{c}^{i_{1} i_{2}}\right) \\
\vdots \\
g^{\prime}\left(x_{i_{1}}^{n}, x_{i_{2}}^{n}, \boldsymbol{c}^{i_{1} i_{2}}\right)
\end{array}\right\} \\
=\left[\begin{array}{ccccc}
\phi_{11}\left(x_{i_{1}}^{1}, x_{i_{2}}^{1}\right) & \cdots & \phi_{1 n}\left(x_{i_{1}}^{1}, x_{i_{2}}^{1}\right) & \cdots & \phi_{n n}\left(x_{i_{1}}^{1}, x_{i_{2}}^{1}\right) \\
\vdots & \vdots & \vdots & \vdots & \vdots \\
\phi_{11}\left(x_{i_{1}}^{1}, x_{i_{2}}^{n}\right) & \cdots & \phi_{1 n}\left(x_{i_{1}}^{1}, x_{i_{2}}^{n}\right) & \cdots & \phi_{n n}\left(x_{i_{1}}^{1}, x_{i_{2}}^{n}\right) \\
\vdots & \vdots & \vdots & \vdots & \vdots \\
\phi_{11}\left(x_{i_{1}}^{n}, x_{i_{2}}^{n}\right) & \cdots & \phi_{1 n}\left(x_{i_{1}}^{n}, x_{i_{2}}^{n}\right) & \cdots & \phi_{n n}\left(x_{i_{1}}^{n}, x_{i_{2}}^{n}\right)
\end{array}\right]^{-1}\left\{\begin{array}{c}
g\left(x_{i_{1}}^{1}, x_{i_{2}}^{1}, \boldsymbol{c}^{i_{1} i_{2}}\right) \\
\vdots \\
g\left(x_{i_{1}}^{1}, x_{i_{2}}^{n}, \boldsymbol{c}^{i_{1} i_{2}}\right) \\
\vdots \\
g\left(x_{i_{1}}^{n}, x_{i_{2}}^{n}, \boldsymbol{c}^{i_{1} i_{2}}\right)
\end{array}\right\} .
\end{gathered}
$$

The interpolation functions $\phi_{j}\left(x_{i}\right)$ and $\phi_{j_{1} j_{2}}\left(x_{i_{1}}, x_{i_{2}}\right)$ can be obtained using the MLS interpolation scheme.

By using (18), $g_{i}\left(x_{i}\right)$ can be generated if $n$ function values are given at corresponding sample points. Similarly, by using $(21), g_{i_{1} i_{2}}\left(x_{i_{1}}, x_{i_{2}}\right)$ can be generated if $n^{2}$ function values at 
corresponding sample points are given. The same procedure shall be repeated for all the firstorder component functions, i.e. $g_{i}\left(x_{i}\right) ; i=1,2, \ldots, N$ and the second-order component functions, i.e. $g_{i_{1} i_{2}}\left(x_{i_{1}}, x_{i_{2}}\right) ; i_{1}, i_{2}=1,2, \ldots, N$.

Step 2: Sum the interpolated values of HDMR expansion terms from zeroth-order to the highest order retained in keeping with the desired accuracy. This leads to first- and secondorder HDMR approximation of the function $g(\boldsymbol{x})$ as

$$
\tilde{g}(\boldsymbol{x})=\sum_{i=1}^{N} \sum_{j=1}^{n} \phi_{j}\left(x_{i}\right) g^{\prime}\left(c_{1}, \ldots, c_{i-1}, x_{i}^{j}, c_{i+1}, \ldots, c_{N}\right)-(N-1) g_{0},
$$

and

$$
\begin{aligned}
\tilde{g}(\boldsymbol{x})= & \sum_{\substack{i_{1}=1, i_{2}=1 \\
i_{1}<i_{2}}}^{N} \sum_{j_{1}=1}^{n} \sum_{j_{2}=1}^{n} \phi_{j_{1} j_{2}}\left(x_{i_{1}}, x_{i_{2}}\right) \\
& \times g^{\prime}\left(c_{1}, \ldots, c_{i_{1}-1}, x_{i_{1}}^{j_{1}}, c_{i_{1}+1}, \ldots, c_{i_{2}-1}, x_{i_{2}}^{j_{2}}, c_{i_{2}+1}, \ldots, c_{N}\right) \\
& -(N-2) \sum_{i=1}^{N} \sum_{j=1}^{n} \phi_{j}\left(x_{i}\right) g^{\prime}\left(c_{1}, \ldots, c_{i-1}, x_{i}^{j}, c_{i+1}, \ldots, c_{N}\right)+\frac{(N-1)(N-2)}{2} g_{0}
\end{aligned}
$$

respectively.

\section{Estimation of failure probability \& sensitivity}

Recall that $\tilde{g}(\boldsymbol{x})$ is the approximate function of the original limit state/performance function. Based on this approximation, let $\hat{\Omega}_{F S}=\{\boldsymbol{x}: \tilde{g}(\boldsymbol{x})<0\}$ define approximate failure set in reliability analysis. Therefore, Monte Carlo method estimates of the failure probability $P_{F}(\theta)$ and its sensitivity $\partial P_{F}(\boldsymbol{\theta}) / \partial \theta_{i}$, employing HDMR approximation are

$$
P_{F}(\boldsymbol{\theta}) \cong E_{\theta}\left[\mathcal{J}_{\hat{\Omega}_{F S}}(\boldsymbol{x})\right]=\lim _{N_{s} \rightarrow \infty} \frac{1}{N_{S}} \sum_{i=1}^{N_{S}} \mathcal{J}_{\hat{\Omega}_{F S}}\left(\boldsymbol{x}^{i}\right),
$$

and

$$
\frac{\partial P_{F}(\boldsymbol{\theta})}{\partial \theta_{i}} \cong E_{\theta}\left[\mathcal{J}_{\hat{\Omega}_{F S}}(\boldsymbol{x}) K_{\theta}^{(1)}(\boldsymbol{x} ; \boldsymbol{\theta})\right]=\lim _{N_{s} \rightarrow \infty} \frac{1}{N_{S}} \sum_{i=1}^{N_{S}} \mathcal{J}_{\hat{\Omega}_{F S}}\left(\boldsymbol{x}^{i}\right) K_{\theta}^{(1)}\left(\boldsymbol{x}^{i} ; \boldsymbol{\theta}\right),
$$

respectively, where $\boldsymbol{x}^{i}$ is $i^{\text {th }}$ realization of $\boldsymbol{x}, N_{S}$ is the sample size, $\mathcal{J}_{\hat{\Omega}_{F S}}\left(\boldsymbol{x}^{i}\right)$ is an indicator of fail or safe state such that

$$
\mathcal{J}_{\hat{\Omega}_{F S}}\left(x^{i}\right)=\left\{\begin{array}{ll}
1, & x^{i} \in \hat{\Omega}_{F S} \\
0, & x^{i} \in \Omega \backslash \hat{\Omega}_{F S}
\end{array} .\right.
$$


Monte Carlo estimate of moment of HDMR approximation can be found as

$$
m_{q}(\boldsymbol{\theta}) \cong E_{\theta}\left[\tilde{g}^{q}(\boldsymbol{x})\right]=\lim _{N_{s} \rightarrow \infty} \frac{1}{N_{S}} \sum_{i=1}^{N_{S}} \tilde{g}^{q}\left(\boldsymbol{x}^{i}\right),
$$

and its sensitivity

$$
\frac{\partial m_{q}(\boldsymbol{\theta})}{\partial \theta_{i}} \cong E_{\theta}\left[\tilde{g}^{q}(\boldsymbol{x}) K_{\theta}^{(1)}(\boldsymbol{x} ; \boldsymbol{\theta})\right]=\lim _{N_{s} \rightarrow \infty} \frac{1}{N_{S}} \sum_{i=1}^{N_{S}} \tilde{g}^{q}\left(\boldsymbol{x}^{i}\right) K_{\theta}^{(1)}\left(\boldsymbol{x}^{i} ; \boldsymbol{\theta}\right) .
$$

Since first- and second-order HDMR approximation leads to explicit representation of the original implicit limit state/performance function, the MCS can be conducted for any sampling size. The total cost of original function evaluation entails a maximum of $(n-1) \times N+1$ and $(n-1)^{2}(N-1) N / 2+(n-1) N+1$ by the present method using first- and second-order HDMR approximation, respectively.

\section{Numerical examples}

Two numerical examples are presented to illustrate the proposed approach, for obtaining firstorder sensitivity of the moment or reliability. Whenever possible, finite-difference method and the direct MCS, is employed to evaluate the accuracy and computational efficiency of the present method. The sample sizes for the direct MCS and the MCS in conjunction with HDMR approximation vary from $10^{4}$ to $10^{6}$, depending on the examples, but they are identical for a specific problem. For first-order HDMR, $n$ equally spaced sample points are deployed along the variable axis through the reference point. Sampling scheme for HDMR approximation of a function that is having one variable $(x)$ and two variables $\left(x_{1}\right.$ and $\left.x_{2}\right)$ using first-order HDMR is shown in figures 1(a) and 1(b) respectively. For second-order HDMR, $n$ equally spaced sample points are deployed along each of the variable axis to form a regular grid. Sampling scheme for HDMR approximation of a function having two variables $\left(x_{1}\right.$ and $\left.x_{2}\right)$ using second-order HDMR is shown in figure 2 . In all numerical examples presented, the reference point $\boldsymbol{c}$ is taken as mean values of the random variables.

\subsection{Example 1: Cubic function with two variables}

Consider a cubic limit state/performance function of the following form:

$$
g(\boldsymbol{x})=2 \cdot 2257-\frac{0 \cdot 025 \sqrt{2}}{27}\left(x_{1}+x_{2}-20\right)^{3}+\frac{33}{140}\left(x_{1}-x_{2}\right),
$$

with two independent normal variables. Mean and standard deviation of the random variables are 10 and 3 , respectively. For evaluating the failure probability $P_{F}$, HDMR approximation is constructed by deploying five equally spaced sample points $(n=5)$ along each of the variable axis. Table 1 compares the results obtained by the present method using HDMR approximation with direct MCS. A sampling size $N_{S}=10^{6}$ is considered in direct MCS to evaluate the failure probability $P_{F}$. Table 1 also contains the computational effort in terms of number of function evaluations, associated with each of the methods. Compared with the failure probability obtained using direct MCS $\left(P_{F}=0.01907\right)$, first- and second-order 


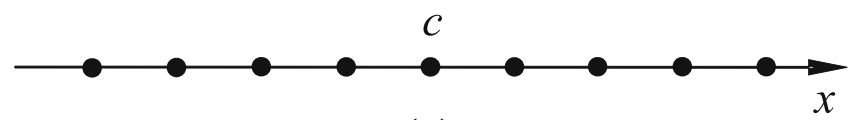

(a)

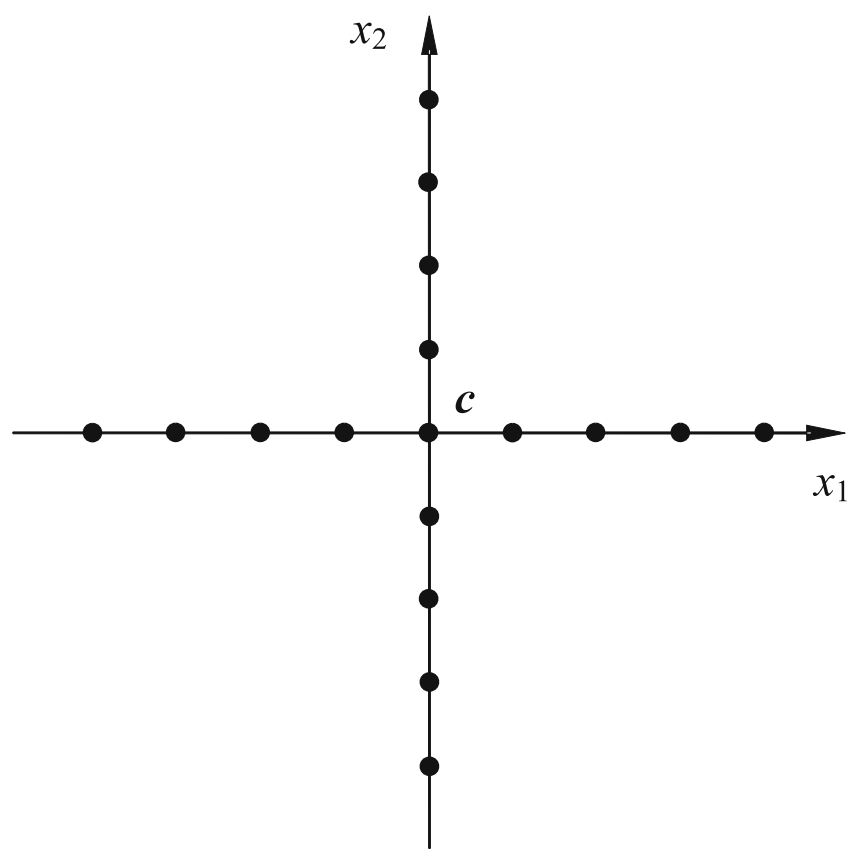

(b)
Figure 1. Sampling scheme for first-order HDMR; (a) for a function having one variable $(x)$; and (b) For a function having two variables $\left(x_{1}\right.$ and $\left.x_{2}\right)$.

HDMR approximation underestimates the failure probability by $16.99 \%\left(P_{F}=0.01583\right)$ and $0.94 \%\left(P_{F}=0.01889\right)$, respectively. Table 2 lists the first-order sensitivities of failure probability with respect to mean and standard deviation of random variables $\partial P_{F} / \partial \mu_{i}$ and $\partial P_{F} / \partial \sigma_{i}$ for $i=1,2$. Similarly, table 3 presents first three moments $m_{q}$ and their first-order sensitivities $\partial m_{q}(\boldsymbol{\theta}) / \partial \mu$ and $\partial m_{q}(\boldsymbol{\theta}) / \partial \sigma$ for $q=1,2,3$. Alternative sensitivity estimates from the finite difference method involving $N_{S}=10^{6}$ samples for each direct simulation run are also calculated, and can be found in the last column of tables 2 and 3 . The results of the proposed approach and the finite difference method agree well.

The effect of number of sample points on first- and second-order HDMR approximation for reliability and sensitivity estimation is studied by carrying out a similar analysis varying $n$ from 3 to 9 . Using first-order HDMR approximation, $P_{F}$ ranges from $0 \cdot 01314(+31 \cdot 11 \%)$ (at $n=3$ ) to $0 \cdot 01569\left(+17.70 \%\right.$ ) (at $n=9$ ), whereas $P_{F}$ ranges from $0 \cdot 01672(+12 \cdot 32 \%$ ) (at $n=3$ ) to $0.01892(+0.79 \%$ ) (at $n=9$ ) using second-order HDMR approximation. Compared with direct MCS, error in the estimated failure probability using different methods is tabulated in table 4. Similarly, errors in the estimated moments and sensitivities are tabulated in tables 5 and 6. It can be observed that, compared with first-order HDMR, second-order approximation resulted in drastic reduction of the approximation error of the estimated failure probability as well as resulted sensitivities. The computational effort in terms of number of function evaluations for second-order HDMR approximation increased from 5 to 9 for $n=3$, 


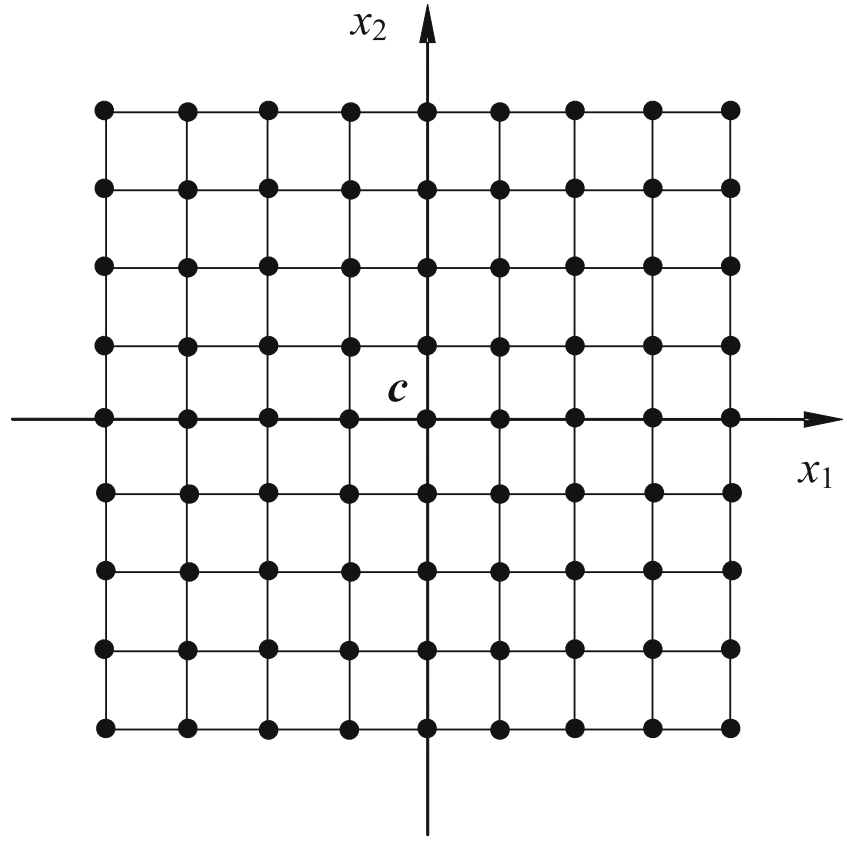

Figure 2. Sampling scheme for second-order HDMR for a function having two variables $\left(x_{1}\right.$ and $\left.x_{2}\right)$.

Table 1. Estimation of error in failure probability using different methods for Example 1.

\begin{tabular}{lcc}
\hline Method & $\begin{array}{c}\text { Failure } \\
\text { probability }\end{array}$ & $\begin{array}{c}\text { Number of function } \\
\text { evaluation }\end{array}$ \\
\hline FORM & $0 \cdot 01302$ & 21 \\
SORM (Hohenbichler et al 1987) & $0 \cdot 01302$ & 204 \\
First-order HDMR & $0 \cdot 01583$ & $9^{(b)}$ \\
Second-order HDMR & $0 \cdot 01889$ & $25^{(c)}$ \\
Direct MCS & $0 \cdot 01907$ & $10^{6}$ \\
\hline
\end{tabular}

(a) Total number of times the original performance function is calculated.

(b) $(n-1) \times N+1=(5-1) \times 2+1=9$

(c) $(n-1)^{2}(N-1) N / 2+(n-1) N+1=(5-1)^{2}(2-1) 2 / 2+(5-1) 2+1=25$

Table 2. Estimation of error in sensitivities of failure probability using different methods for Example 1.

\begin{tabular}{lrrr}
\hline & First-order HDMR & Second-order HDMR & Direct MCS ${ }^{(a)}$ \\
\hline$\partial P_{F} / \partial \mu_{1}$ & $-5.56 \times 10^{-3}$ & $-5.06 \times 10^{-3}$ & $-5.07 \times 10^{-3}$ \\
$\partial P_{F} / \partial \mu_{2}$ & $4.38 \times 10^{-3}$ & $1.24 \times 10^{-2}$ & $1.36 \times 10^{-2}$ \\
$\partial P_{F} / \partial \sigma_{1}$ & $1.19 \times 10^{-2}$ & $2.63 \times 10^{-2}$ & $2.67 \times 10^{-2}$ \\
$\partial P_{F} / \partial \sigma_{2}$ & $2.32 \times 10^{-2}$ & $2.46 \times 10^{-2}$ & $2.48 \times 10^{-2}$ \\
\hline
\end{tabular}

${ }^{(a)}$ For sensitivity estimation, finite difference with $1 \%$ perturbation is used 
Table 3. Moments and sensitivities of moments (Example 1).

\begin{tabular}{|c|c|c|c|}
\hline & First-order HDMR & Second-order HDMR & Direct $\operatorname{MCS}^{(a)}$ \\
\hline$m_{1}$ & $2 \cdot 2270$ & $2 \cdot 2245$ & $2 \cdot 2245$ \\
\hline$m_{2}$ & $6 \cdot 0002$ & $6 \cdot 1106$ & $6 \cdot 1088$ \\
\hline$m_{3}$ & 17.9941 & $18 \cdot 7491$ & $18 \cdot 7502$ \\
\hline$\partial m_{1} / \partial \mu_{1}$ & 0.2008 & $0 \cdot 1633$ & $0 \cdot 1632$ \\
\hline$\partial m_{2} / \partial \mu_{1}$ & 0.8918 & 0.7211 & 0.7215 \\
\hline$\partial m_{3} / \partial \mu_{1}$ & $3 \cdot 5453$ & 2.7445 & 2.7448 \\
\hline$\partial m_{1} / \partial \mu_{2}$ & $-0 \cdot 2725$ & $-0 \cdot 3082$ & $-0 \cdot 3082$ \\
\hline$\partial m_{2} / \partial \mu_{2}$ & $-1 \cdot 2125$ & $-1 \cdot 3826$ & $-1 \cdot 3827$ \\
\hline$\partial m_{3} / \partial \mu_{2}$ & -5.0903 & $-5 \cdot 9408$ & $-5 \cdot 9410$ \\
\hline$\partial m_{1} / \partial \sigma_{1}$ & $0 \cdot 0004$ & $0 \cdot 0002$ & $0 \cdot 0002$ \\
\hline$\partial m_{2} / \partial \sigma_{1}$ & 0.1713 & -0.2911 & -0.2912 \\
\hline$\partial m_{3} / \partial \sigma_{1}$ & $1 \cdot 1388$ & -1.9266 & -1.9267 \\
\hline$\partial m_{1} / \partial \sigma_{2}$ & $-8.3321 \times 10^{-4}$ & 0.0002 & 0.0002 \\
\hline$\partial m_{2} / \partial \sigma_{2}$ & 0.5792 & -0.7211 & -0.7210 \\
\hline$\partial m_{3} / \partial \sigma_{2}$ & $3 \cdot 8612$ & $-4 \cdot 8139$ & $-4 \cdot 8140$ \\
\hline
\end{tabular}

${ }^{(a)}$ For sensitivity estimation, finite difference with $1 \%$ perturbation is used

Table 4. Estimation of error in failure probability using different methods for Example 1.

\begin{tabular}{lcccc}
\hline & \multicolumn{4}{c}{ Number of sample points, $n$} \\
\cline { 2 - 5 } Method & 3 & 5 & \multicolumn{1}{c}{7} & 9 \\
\hline First-order HDMR & $31.11 \%$ & $16.99 \%$ & $16.66 \%$ & $17.70 \%$ \\
Second-order HDMR & $12.32 \%$ & $0.94 \%$ & $0.52 \%$ & $0.79 \%$ \\
\hline
\end{tabular}

Table 5. Estimation of error in sensitivities of failure probability using different methods for Example 1.

\begin{tabular}{|c|c|c|c|c|c|}
\hline \multirow[b]{2}{*}{ Method } & & \multicolumn{4}{|c|}{ Number of sample points, $n$} \\
\hline & & 3 & 5 & 7 & 9 \\
\hline First-order & $\begin{array}{l}\partial P_{F} / \partial \mu_{1} \\
\partial P_{F} / \partial \mu_{2} \\
\partial P_{F} / \partial \sigma_{1} \\
\partial P_{F} / \partial \sigma_{2}\end{array}$ & $\begin{array}{r}-49.57 \\
16 \cdot 29 \\
68 \cdot 74 \\
44.55\end{array}$ & $\begin{array}{r}-9.66 \\
67.79 \\
55.43 \\
6.45\end{array}$ & $\begin{array}{r}-11.44 \\
66.82 \\
55.18 \\
6.98\end{array}$ & $\begin{array}{r}-10 \cdot 26 \\
67 \cdot 06 \\
55 \cdot 81 \\
7 \cdot 74\end{array}$ \\
\hline Second-order & $\begin{array}{l}\partial P_{F} / \partial \mu_{1} \\
\partial P_{F} / \partial \mu_{2} \\
\partial P_{F} / \partial \sigma_{1} \\
\partial P_{F} / \partial \sigma_{2}\end{array}$ & $\begin{array}{r}-5 \cdot 72 \\
15 \cdot 15 \\
7 \cdot 12 \\
6 \cdot 45\end{array}$ & $\begin{array}{l}0 \cdot 20 \\
8 \cdot 82 \\
1.50 \\
0 \cdot 81\end{array}$ & $\begin{array}{r}-0.20 \\
5.15 \\
-0.75 \\
0.00\end{array}$ & $\begin{array}{r}-1.18 \\
6.62 \\
0.37 \\
2.82\end{array}$ \\
\hline
\end{tabular}


Table 6. Estimation of error in moments and sensitivities of moments using different methods for Example 1.

\begin{tabular}{|c|c|c|c|c|c|}
\hline \multirow[b]{2}{*}{ Method } & & \multicolumn{4}{|c|}{ Number of sample points, $n$} \\
\hline & & 3 & 5 & 7 & 9 \\
\hline \multirow{15}{*}{ First-order } & $m_{1}$ & $-0 \cdot 11$ & $-0 \cdot 11$ & $-0 \cdot 11$ & $-0 \cdot 11$ \\
\hline & $m_{2}$ & $2 \cdot 38$ & 1.78 & $1 \cdot 76$ & $1 \cdot 81$ \\
\hline & $m_{3}$ & $5 \cdot 33$ & 4.03 & 3.99 & 4.11 \\
\hline & $\partial m_{1} / \partial \mu_{1}$ & -37.93 & $-23 \cdot 04$ & $-23 \cdot 65$ & -23.65 \\
\hline & $\partial m_{2} / \partial \mu_{1}$ & $-39 \cdot 02$ & -23.60 & $-24 \cdot 21$ & $-24 \cdot 23$ \\
\hline & $\partial m_{3} / \partial \mu_{1}$ & $-46 \cdot 24$ & $-29 \cdot 16$ & $-29 \cdot 87$ & -29.79 \\
\hline & $\partial m_{1} / \partial \mu_{2}$ & $19 \cdot 44$ & 11.58 & 11.68 & 11.91 \\
\hline & $\partial m_{2} / \partial \mu_{2}$ & $20 \cdot 11$ & $12 \cdot 31$ & $12 \cdot 40$ & 12.63 \\
\hline & $\partial m_{3} / \partial \mu_{2}$ & $25 \cdot 33$ & $14 \cdot 32$ & $14 \cdot 56$ & $14 \cdot 78$ \\
\hline & $\partial m_{1} / \partial \sigma_{1}$ & $-125 \cdot 29$ & $-100 \cdot 00$ & $-92 \cdot 70$ & -92.94 \\
\hline & $\partial m_{2} / \partial \sigma_{1}$ & $-4 \cdot 36$ & $41 \cdot 17$ & $40 \cdot 01$ & $40 \cdot 01$ \\
\hline & $\partial m_{3} / \partial \sigma_{1}$ & -5.06 & $40 \cdot 89$ & 39.69 & $39 \cdot 69$ \\
\hline & $\partial m_{1} / \partial \sigma_{2}$ & $-334 \cdot 36$ & $-316 \cdot 61$ & $-306 \cdot 92$ & -317.58 \\
\hline & $\partial m_{2} / \partial \sigma_{2}$ & 48.96 & 19.67 & $20 \cdot 57$ & $20 \cdot 75$ \\
\hline & $\partial m_{3} / \partial \sigma_{2}$ & $48 \cdot 77$ & $19 \cdot 79$ & $20 \cdot 59$ & $20 \cdot 86$ \\
\hline \multirow{15}{*}{ Second-order } & $m_{1}$ & -0.01 & $0 \cdot 00$ & $0 \cdot 00$ & $0 \cdot 00$ \\
\hline & $m_{2}$ & -0.04 & -0.03 & $-0 \cdot 01$ & -0.02 \\
\hline & $m_{3}$ & $0 \cdot 01$ & 0.01 & $0 \cdot 00$ & $0 \cdot 00$ \\
\hline & $\partial m_{1} / \partial \mu_{1}$ & -0.43 & -0.06 & -0.06 & $-0 \cdot 18$ \\
\hline & $\partial m_{2} / \partial \mu_{1}$ & -0.04 & 0.06 & $0 \cdot 01$ & 0.04 \\
\hline & $\partial m_{3} / \partial \mu_{1}$ & 0.02 & $0 \cdot 01$ & $0 \cdot 00$ & 0.00 \\
\hline & $\partial m_{1} / \partial \mu_{2}$ & 0.26 & $0 \cdot 00$ & -0.06 & 0.00 \\
\hline & $\partial m_{2} / \partial \mu_{2}$ & -0.04 & $0 \cdot 01$ & -0.01 & 0.01 \\
\hline & $\partial m_{3} / \partial \mu_{2}$ & $-0 \cdot 01$ & $0 \cdot 00$ & $0 \cdot 00$ & 0.00 \\
\hline & $\partial m_{1} / \partial \sigma_{1}$ & $-50 \cdot 00$ & $0 \cdot 00$ & $5 \cdot 00$ & 0.00 \\
\hline & $\partial m_{2} / \partial \sigma_{1}$ & $-0 \cdot 14$ & 0.03 & $0 \cdot 10$ & 0.03 \\
\hline & $\partial m_{3} / \partial \sigma_{1}$ & 0.03 & $0 \cdot 01$ & $0 \cdot 01$ & 0.01 \\
\hline & $\partial m_{1} / \partial \sigma_{2}$ & $10 \cdot 00$ & 0.00 & 0.00 & 0.00 \\
\hline & $\partial m_{2} / \partial \sigma_{2}$ & $-0 \cdot 10$ & -0.01 & -0.01 & -0.01 \\
\hline & $\partial m_{3} / \partial \sigma_{2}$ & 0.02 & 0.00 & 0.01 & 0.00 \\
\hline
\end{tabular}

from 9 to 25 for $n=5$, from 13 to 49 for $n=7$, and from 17 to 81 for $n=9$, when compared with first-order HDMR approximation.

\subsection{Example 2: Vibration of 6-DOF system}

This example considers a four-story building excited by a single period sinusoidal pulse of ground motion, studied by Gavin \& Yau (2008). Figure 3a shows the four-storey building with isolation systems and figure $3 \mathrm{~b}$ presents the acceleration history. The building contains isolated equipment resting on the second floor. The motion of the ground floor is resisted mainly by base isolation bearings (Wen 1976) and if its displacement exceeds $D_{c}(=0.50 \mathrm{~m})$ then an additional stiffness force contributes to the resistance. Mass, stiffness and damping coefficient $m_{f}, k_{f}$ and $c_{f}$, respectively at each floor are assumed to be same.

There are two isolated masses, representing isolated, shock-sensitive equipment resting on the second floor. The larger mass $m_{1}(=500 \mathrm{~kg})$ is connected to the floor by a relatively 


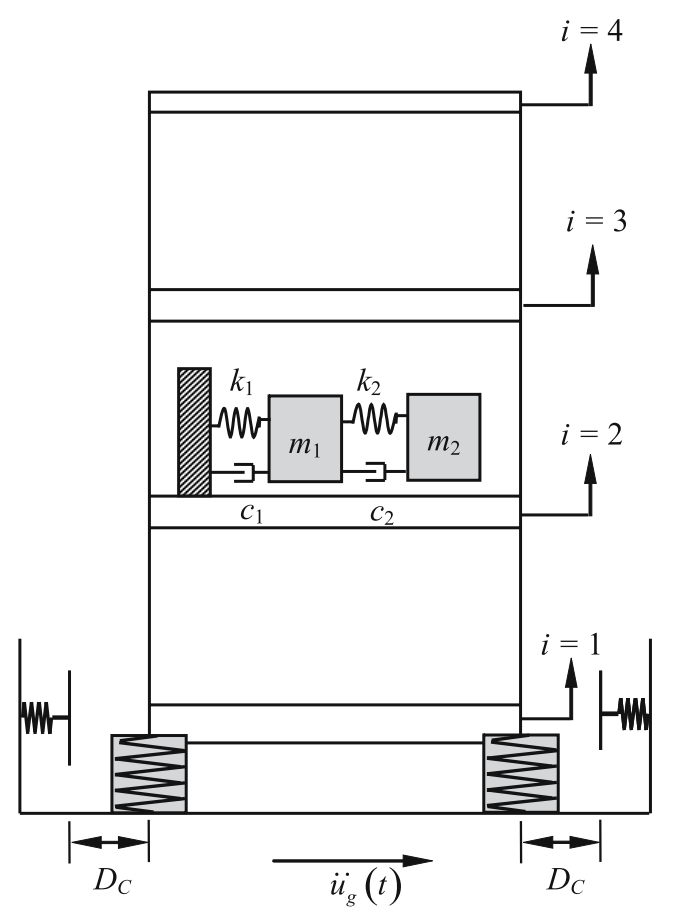

(a)

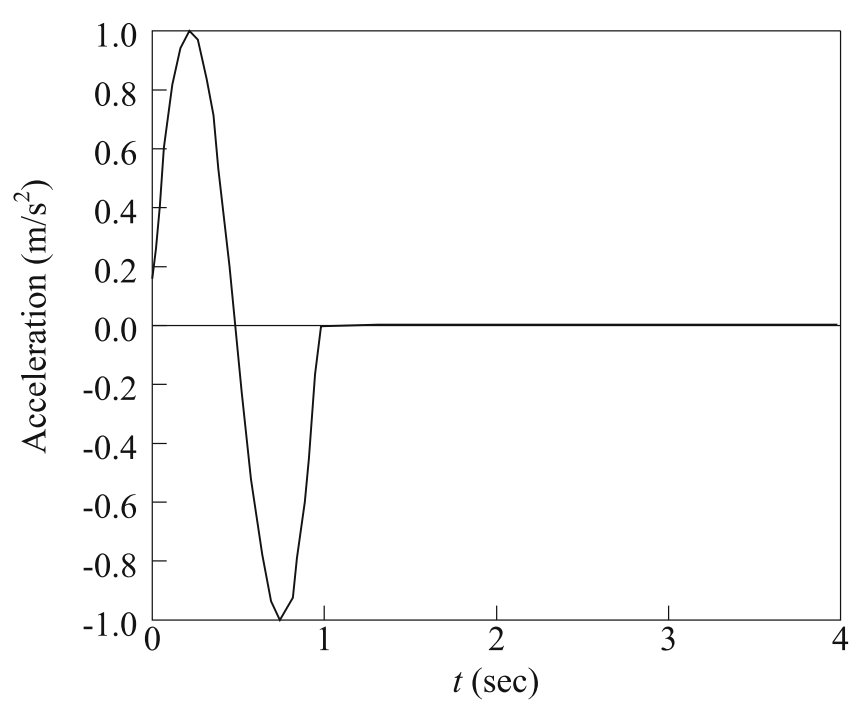

(b)
Figure 3. Problem statement (Example 2); (a) Base isolated structure with an equipment isolation system on the second floor; and (b) Acceleration history.

flexible spring, $k_{1}(=2500 \mathrm{~N} / \mathrm{m})$, and a damper, $c_{1}(=350 \mathrm{~N} / \mathrm{m} / \mathrm{s})$, representing the isolation system. The smaller mass $\left(m_{2}=100 \mathrm{~kg}\right)$ is connected to the larger mass by a relatively stiff spring, $k_{2}\left(=10^{5} \mathrm{~N} / \mathrm{m}\right)$, and a damper, $c_{2}(=200 \mathrm{~N} / \mathrm{m} / \mathrm{s})$, representing the equipment itself. All variables are assumed to be lognormal and independent. Probabilistic descriptions of the random variables are listed in table 7 . The limit state/performance function is defined by the 
Table 7. Properties of the random variables for Example 2.

\begin{tabular}{llllcl}
\hline $\begin{array}{l}\text { Random } \\
\text { variable }\end{array}$ & Distribution & Units & \multicolumn{1}{c}{ Description } & Mean & COV \\
\hline$m_{f}$ & Lognormal & $\mathrm{kg}$ & Floor mass & 6000 & $0 \cdot 10$ \\
$k_{f}$ & Lognormal & $\mathrm{N} / \mathrm{m}$ & Floor stiffness & $3 \times 10^{7}$ & $0 \cdot 10$ \\
$c_{f}$ & Lognormal & $\mathrm{N} / \mathrm{m} / \mathrm{s}$ & Floor damping coefficient & $6 \times 10^{4}$ & $0 \cdot 20$ \\
$f_{y}$ & Lognormal & $\mathrm{N}$ & Isolation yield force & $2 \times 10^{4}$ & $0 \cdot 20$ \\
$d_{y}$ & Lognormal & $\mathrm{m}$ & Isolation yield displacement & $0 \cdot 05$ & $0 \cdot 20$ \\
$k_{c}$ & Lognormal & $\mathrm{N} / \mathrm{m}$ & Isolation contact stiffness & $3 \times 10^{7}$ & $0 \cdot 30$ \\
$T$ & Lognormal & $\mathrm{s}$ & Force period & $1 \cdot 0$ & $0 \cdot 20$ \\
$A$ & Lognormal & $\mathrm{m} / \mathrm{m} / \mathrm{s}$ & Force amplitude & $1 \cdot 0$ & $0 \cdot 50$ \\
\hline
\end{tabular}

combination of three failure modes leading to system failure and is the following form

$$
\begin{aligned}
g(\boldsymbol{x})= & 12 \cdot 50\left(0.04-\max _{t}\left|x_{f_{i}}(t)-x_{f_{i-1}}(t)\right|\right)_{i=2,3,4} \\
& +\left(0.50-\max _{t}\left|\ddot{u}_{g}(t)+\ddot{x}_{m_{2}}(t)\right|\right) \\
& +2 \cdot 0\left(0.25-\max _{t}\left|x_{f_{2}}(t)-x_{m_{1}}(t)\right|\right),
\end{aligned}
$$

where $x_{f_{i}}(t)$ refers to the displacement of $i^{\text {th }}$ floor and $\left(x_{f_{i}}(t)-x_{f_{i-1}}(t)\right)$ is the inter storey drift. $\ddot{u}_{g}(t)$ is the ground acceleration and $\ddot{x}_{m_{2}}(t)$ is the acceleration smaller mass block. The displacement $x_{m_{1}}(t)$ is of the larger mass block, and represents the displacement of the equipment isolation system. The limit state/performance function in (31) is the overall representation of three failure modes. The first term describes the damage to the structural system due to excessive deformation. The second term represents the damage to equipment caused by excessive acceleration. The last term represents the damage of the isolation system. The weighing factors, multiplied with each term in (31), are mainly to emphasize the equal contribution of the individual failure modes to the overall failure of system. It is desirable that (a) inter storey drift is limited to $0.04 \mathrm{~m}$, (b) the peak acceleration of the equipment is less than $0.5 \mathrm{~m} / \mathrm{s}^{2}$, and (c) the displacement across the equipment isolation system is less than $0.25 \mathrm{~m}$. Equation (31) signifies overall system failure, which does not necessarily occur when above mentioned one or two failure criteria satisfies. For evaluating the failure probability $P_{F}$, first-order HDMR approximation is constructed by deploying five equally spaced sample points $(n=5)$ along each of the variable axis. The reference point is taken as mean values of the random variables. Table 8 compares the results obtained using first- and second-order HDMR approximation with FORM, SORM (Hohenbichler et al 1987), and direct MCS and also presents the computational effort in terms of number of function evaluations, associated with each of the methods. The benchmark solution of the failure probability is obtained by direct MCS with $N_{S}=10^{5}$. Compared with the benchmark solution $\left(P_{F}=0 \cdot 19599\right)$, FORM and SORM overestimates the failure probability by around $15.05 \%\left(P_{F}=0.22549\right)$ and $8.46 \%\left(P_{F}=0.21410\right)$, respectively. First-order HDMR approximation overestimates the failure probability by about $1.88 \%\left(P_{F}=0.19968\right)$ and it needs only 33 function evaluations, while FORM, SORM and direct MCS requires 86,356 and $10^{5}$ number of original function evaluations, respectively. This shows the accuracy and the efficiency (in terms of original 
Table 8. Estimation of failure probability for Example 2.

\begin{tabular}{lcc}
\hline Method & $\begin{array}{c}\text { Failure } \\
\text { probability }\end{array}$ & $\begin{array}{c}\text { Number of function } \\
\text { evaluation }^{(a)}\end{array}$ \\
\hline FORM & $0 \cdot 22549$ & 86 \\
SORM (Hohenbichler et al 1987) & $0 \cdot 21410$ & 357 \\
First-order HDMR & $0 \cdot 19968$ & $33^{(b)}$ \\
Second-order HDMR & $0 \cdot 19246$ & $481^{(c)}$ \\
Direct MCS & $0 \cdot 19599$ & $10^{5}$ \\
\hline
\end{tabular}

${ }^{(a)}$ Total number of times the original performance function is calculated.

(b) $(n-1) \times N+1=(5-1) \times 8+1=33$

${ }^{(c)}(n-1)^{2}(N-1) N / 2+(n-1) N+1=(5-1)^{2}(8-1) 8 / 2+(5-1) 8+1=481$

function calculations) of the first-order HDMR approximation, over FORM, SORM and direct MCS. Compared to the first-order HDMR, the error in the estimated failure probability reduces from $+1.88 \%$ to $-1.80 \%$, and the number of function evaluations increases from 33 to 481 , using second-order HDMR approximation.

Tables 9 and 10 present the first-order sensitivities of failure probability with respect to mean and standard deviation of random variables $\partial P_{F} / \partial \mu_{i}$ and $\partial P_{F} / \partial \sigma_{i}$ for $i=1, \ldots, 8$,

Table 9. Sensitivities of failure probability with respect to mean (Example 2).

\begin{tabular}{lccc}
\hline & First-order HDMR & Second-order HDMR & Direct MCS $^{(a)}$ \\
\hline$\partial P_{F} / \partial \mu_{1}$ & $-7.8376 \times 10^{-6}$ & $-8.0783 \times 10^{-6}$ & $-8.2371 \times 10^{-6}$ \\
$\partial P_{F} / \partial \mu_{2}$ & $-5.9548 \times 10^{-10}$ & $-6.1156 \times 10^{-10}$ & $-6.1242 \times 10^{-10}$ \\
$\partial P_{F} / \partial \mu_{3}$ & $9.1605 \times 10^{-8}$ & $8.9812 \times 10^{-8}$ & $8.9905 \times 10^{-8}$ \\
$\partial P_{F} / \partial \mu_{4}$ & 5.4109 & 5.4219 & 5.4309 \\
$\partial P_{F} / \partial \mu_{5}$ & $5.2208 \times 10^{-6}$ & $5.6898 \times 10^{-6}$ & $5.7218 \times 10^{-6}$ \\
$\partial P_{F} / \partial \mu_{6}$ & $6.5064 \times 10^{-11}$ & $6.9064 \times 10^{-11}$ & $6.9064 \times 10^{-11}$ \\
$\partial P_{F} / \partial \mu_{7}$ & 0.9655 & 0.9788 & 0.9834 \\
$\partial P_{F} / \partial \mu_{8}$ & 0.3705 & 0.4186 & 0.4215 \\
\hline
\end{tabular}

${ }^{(a)}$ For sensitivity estimation, finite difference with $1 \%$ perturbation is used

Table 10. Sensitivities of failure probability with respect to standard deviation (Example 2).

\begin{tabular}{lccc}
\hline & First-order HDMR & Second-order HDMR & Direct MCS \\
\hline$\partial P_{F} / \partial \sigma_{1}$ & $-5.0739 \times 10^{-6}$ & $-5.1682 \times 10^{-6}$ & $-5.1702 \times 10^{-6}$ \\
$\partial P_{F} / \partial \sigma_{2}$ & $-1.9825 \times 10^{-10}$ & $-1.9961 \times 10^{-10}$ & $-1.9965 \times 10^{-10}$ \\
$\partial P_{F} / \partial \sigma_{3}$ & $1.2518 \times 10^{-7}$ & $1.3112 \times 10^{-7}$ & $1.3117 \times 10^{-7}$ \\
$\partial P_{F} / \partial \sigma_{4}$ & -0.7237 & -0.7338 & -0.7342 \\
$\partial P_{F} / \partial \sigma_{5}$ & $-4.4779 \times 10^{-6}$ & $-4.4887 \times 10^{-6}$ & $-4.4878 \times 10^{-6}$ \\
$\partial P_{F} / \partial \sigma_{6}$ & $1.3931 \times 10^{-10}$ & $1.3941 \times 10^{-10}$ & $1.3942 \times 10^{-10}$ \\
$\partial P_{F} / \partial \sigma_{7}$ & 0.4060 & 0.4106 & 0.4106 \\
$\partial P_{F} / \partial \sigma_{8}$ & $-6.4636 \times 10^{-3}$ & $-6.4736 \times 10^{-3}$ & $-6.4737 \times 10^{-3}$ \\
\hline
\end{tabular}

${ }^{(a)}$ For sensitivity estimation, finite difference with $1 \%$ perturbation is used 
Table 11. Moments and sensitivities of moments (Example 1).

\begin{tabular}{|c|c|c|c|}
\hline & First-order HDMR & Second-order HDMR & Direct $\operatorname{MCS}^{(a)}$ \\
\hline$m_{1}$ & 0.3741 & $0 \cdot 3717$ & 0.3722 \\
\hline$m_{2}$ & 0.3358 & 0.3347 & 0.3354 \\
\hline$m_{3}$ & $0 \cdot 2615$ & $0 \cdot 2602$ & $0 \cdot 2613$ \\
\hline$\partial m_{1} / \partial \mu_{1}$ & $1.3873 \times 10^{-5}$ & $1.4615 \times 10^{-5}$ & $1.4621 \times 10^{-5}$ \\
\hline$\partial m_{1} / \partial \mu_{2}$ & $2.9342 \times 10^{-10}$ & $1.3468 \times 10^{-9}$ & $1.3476 \times 10^{-9}$ \\
\hline$\partial m_{1} / \partial \mu_{3}$ & $-6.0250 \times 10^{-8}$ & $-1.2104 \times 10^{-7}$ & $-1.2107 \times 10^{-7}$ \\
\hline$\partial m_{1} / \partial \mu_{4}$ & -9.4181 & -9.7674 & -9.7682 \\
\hline$\partial m_{1} / \partial \mu_{5}$ & $-9.1614 \times 10^{-6}$ & $-8.9295 \times 10^{-6}$ & $-8.9308 \times 10^{-6}$ \\
\hline$\partial m_{1} / \partial \mu_{6}$ & $-2.2457 \times 10^{-11}$ & $-1.7345 \times 10^{-10}$ & $-1.7385 \times 10^{-10}$ \\
\hline$\partial m_{1} / \partial \mu_{7}$ & -1.5725 & -1.5691 & -1.5708 \\
\hline$\partial m_{1} / \partial \mu_{8}$ & -0.7712 & -0.7722 & -0.7735 \\
\hline$\partial m_{1} / \partial \sigma_{1}$ & $8.9684 \times 10^{-6}$ & $9 \cdot 5177 \times 10^{-6}$ & $9.5198 \times 10^{-6}$ \\
\hline$\partial m_{1} / \partial \sigma_{2}$ & $-4.9191 \times 10^{-11}$ & $-1.3686 \times 10^{-9}$ & $-1.3716 \times 10^{-9}$ \\
\hline$\partial m_{1} / \partial \sigma_{3}$ & $-1 \cdot 1677 \times 10^{-7}$ & $1.3160 \times 10^{-7}$ & $1.3271 \times 10^{-7}$ \\
\hline$\partial m_{1} / \partial \sigma_{4}$ & 2.5100 & 2.9250 & 2.9269 \\
\hline$\partial m_{1} / \partial \sigma_{5}$ & $8 \cdot 1566 \times 10^{-6}$ & $8.8671 \times 10^{-6}$ & $8.8689 \times 10^{-6}$ \\
\hline$\partial m_{1} / \partial \sigma_{6}$ & $-1.7508 \times 10^{-10}$ & $-4 \cdot 1060 \times 10^{-10}$ & $-4.1108 \times 10^{-10}$ \\
\hline$\partial m_{1} / \partial \sigma_{7}$ & 0.1116 & 0.1019 & $0 \cdot 1027$ \\
\hline$\partial m_{1} / \partial \sigma_{8}$ & 0.2909 & 0.2966 & 0.2974 \\
\hline
\end{tabular}

${ }^{(a)}$ For sensitivity estimation, finite difference with $1 \%$ perturbation is used

respectively. Table 11 lists the first three moments $m_{q}$ and their first-order sensitivities $\partial m_{1}(\boldsymbol{\theta}) / \partial \mu_{i}$ and $\partial m_{1}(\boldsymbol{\theta}) / \partial \sigma_{i}$ for $i=1, \ldots, 8$. The agreement between the results of proposed approach and the exact solution demonstrates that the proposed approach can indeed

Table 12. Estimation of error in failure probability and sensitivities of failure probability using different methods for Example 2.

\begin{tabular}{lrc}
\hline & First-order & Second-order \\
\hline$P_{F}$ & -1.883 & 1.801 \\
$\partial P_{F} / \partial \mu_{1}$ & 4.850 & 1.928 \\
$\partial P_{F} / \partial \mu_{2}$ & 2.766 & 0.140 \\
$\partial P_{F} / \partial \mu_{3}$ & -1.891 & 0.103 \\
$\partial P_{F} / \partial \mu_{4}$ & 0.368 & 0.166 \\
$\partial P_{F} / \partial \mu_{5}$ & 8.756 & 0.559 \\
$\partial P_{F} / \partial \mu_{6}$ & 5.792 & 0.000 \\
$\partial P_{F} / \partial \mu_{7}$ & 1.820 & 0.468 \\
$\partial P_{F} / \partial \mu_{8}$ & 12.100 & 0.688 \\
$\partial P_{F} / \partial \sigma_{1}$ & 1.863 & 0.039 \\
$\partial P_{F} / \partial \sigma_{2}$ & 0.701 & 0.020 \\
$\partial P_{F} / \partial \sigma_{3}$ & 4.567 & 0.038 \\
$\partial P_{F} / \partial \sigma_{4}$ & 1.430 & 0.054 \\
$\partial P_{F} / \partial \sigma_{5}$ & 0.221 & -0.020 \\
$\partial P_{F} / \partial \sigma_{6}$ & 0.079 & 0.007 \\
$\partial P_{F} / \partial \sigma_{7}$ & 1.120 & 0.000 \\
$\partial P_{F} / \partial \sigma_{8}$ & 0.156 & 0.002 \\
\hline
\end{tabular}


Table 13. Estimation of error in moments and sensitivities of moments using different methods for Example 2.

\begin{tabular}{lrc}
\hline & First-order & Second-order \\
\hline$m_{1}$ & -0.510 & $0 \cdot 134$ \\
$m_{2}$ & $-0 \cdot 119$ & $0 \cdot 209$ \\
$m_{3}$ & $-0 \cdot 077$ & $0 \cdot 421$ \\
$\partial m_{1} / \partial \mu_{1}$ & $5 \cdot 116$ & $0 \cdot 041$ \\
$\partial m_{1} / \partial \mu_{2}$ & $78 \cdot 226$ & $0 \cdot 059$ \\
$\partial m_{1} / \partial \mu_{3}$ & $50 \cdot 235$ & $0 \cdot 025$ \\
$\partial m_{1} / \partial \mu_{4}$ & $3 \cdot 584$ & $0 \cdot 008$ \\
$\partial m_{1} / \partial \mu_{5}$ & -2.582 & $0 \cdot 015$ \\
$\partial m_{1} / \partial \mu_{6}$ & $87 \cdot 083$ & $0 \cdot 230$ \\
$\partial m_{1} / \partial \mu_{7}$ & $-0 \cdot 108$ & $0 \cdot 108$ \\
$\partial m_{1} / \partial \mu_{8}$ & $0 \cdot 297$ & $0 \cdot 168$ \\
$\partial m_{1} / \partial \sigma_{1}$ & $5 \cdot 792$ & $0 \cdot 022$ \\
$\partial m_{1} / \partial \sigma_{2}$ & 96.414 & $0 \cdot 219$ \\
$\partial m_{1} / \partial \sigma_{3}$ & 187.989 & $0 \cdot 836$ \\
$\partial m_{1} / \partial \sigma_{4}$ & $14 \cdot 244$ & $0 \cdot 065$ \\
$\partial m_{1} / \partial \sigma_{5}$ & 8.031 & $0 \cdot 020$ \\
$\partial m_{1} / \partial \sigma_{6}$ & $57 \cdot 410$ & $0 \cdot 117$ \\
$\partial m_{1} / \partial \sigma_{7}$ & -8.666 & $0 \cdot 779$ \\
$\partial m_{1} / \partial \sigma_{8}$ & $2 \cdot 186$ & $0 \cdot 269$ \\
\hline
\end{tabular}

account high nonlinearity and large input uncertainties. Similar to the Example 1, alternative sensitivity estimates from the finite difference method are also calculated, and can be found in the last column of tables 9-11. The agreement between the results of the proposed method and the finite difference method is good. Compared with direct MCS, errors in the estimated failure probability and sensitivities of failure probability using different methods are tabulated in table 12. Similarly, errors in the estimated moments and sensitivities are tabulated in table 13. It can be observed that, compared with first-order HDMR, second-order approximation resulted in drastic reduction of the approximation error of the estimated failure probability as well as resulted sensitivities.

\section{Conclusions}

This paper presented a new computational method based on HDMR and score functions associated with the probability distribution of a random input, for estimating stochastic sensitivities of structural/mechanical systems with respect to probability distribution parameters. Both the probabilistic response and its sensitivities can be estimated from a single stochastic analysis, without requiring limit state/performance function gradients. The effort in obtaining probabilistic sensitivities can be viewed as calculating the conditional response at a selected deterministic input, defined by sample points. Therefore, the proposed method can be easily adapted for solving stochastic problems involving third-party, commercial FE codes. First- and second-order HDMR approximation are employed to solve two numerical problems, where the performance functions are linear or nonlinear, include Gaussian and/or non-Gaussian random variables, and are described by simple mathematical functions or mechanical responses from finite-element analysis. The results indicate that HDMR approximation, in particular the second-order, provide very accurate estimates of sensitivities of statistical moments or 
reliability. The computational effort by the first-order HDMR approximation varies linearly with respect to the number of random variables, and therefore first-order HDMR is economical. In contrast, second-order HDMR approximation, which generally outperforms first-order HDMR approximation, demands a quadratic cost scaling, making it more expensive than first-order approximation. Nonetheless, both approaches are far less expensive than the finitedifference method or the existing score function method entailing direct MCS.

It can be argued from the discussion of the formulation that, the proposed methodology can be applied to solve any multi-physics problems. Some of the work, in the field of stochastic electromagnetic and flow-structure interaction, is underway.

The authors would like to acknowledge the financial support by the Board of Research in Nuclear Sciences, India (2004/36/39-BRNS/2332). First author acknowledges the support of the Royal Society through Newton International Fellowship.

\section{Nomenclature}

$\begin{array}{ll}E_{\boldsymbol{\theta}} & \text { Expectation operator } \\ \Omega_{F} & \text { Failure set } \\ \hat{\Omega}_{F S}=\{\boldsymbol{x}: \tilde{g}(\boldsymbol{x})<0\} & \text { Approximate failure set } \\ \mathcal{J}_{\Omega_{F}} & \text { Indicator of fail or safe set } \\ K_{\theta}^{(1)}(\boldsymbol{x} ; \boldsymbol{\theta}) & \text { First-order score function for the parameter } \theta \\ \mu_{i} & \text { Mean of input random variable, } i \\ \varphi_{j}\left(x_{i}\right), \varphi_{j_{1} j_{2}}\left(x_{i_{1}}, x_{i_{2}}\right) & \text { Moving least square interpolation function } \\ \rho_{i j} & \text { Correlation coefficient between } x_{i} \text { and } x_{j} \\ \sigma_{i} & \text { Standard deviation of input random variable, } i \\ \Re & \text { Real coordinate space } \\ \Sigma & \text { Covariance matrix } \\ f_{\boldsymbol{x}}(\boldsymbol{x} ; \boldsymbol{\theta}) & \text { Distribution function of } \boldsymbol{x} \text { with parameters } \boldsymbol{\theta} \\ g(\boldsymbol{x}) & \text { Limit state/performance function } \\ \tilde{g}(\boldsymbol{x}) & \text { Approximation of original limit state/performance function, } g(\boldsymbol{x}) \\ g_{i}\left(x_{i}\right) & \text { First-order HDMR component function } \\ g_{i_{1} i_{2}}\left(x_{i_{1}}, x_{i_{2}}\right) & \text { Second-order HDMR component function } \\ h(\boldsymbol{\theta}) & \text { Generic probabilistic response } \\ P_{F}(\boldsymbol{\theta}) & \text { Failure probability } \\ \partial P_{F}(\boldsymbol{\theta}) / \partial \theta_{i} & \text { Sensitivity of failure probability with respect to the parameter } \theta_{i} \\ \partial m_{q}(\boldsymbol{\theta}) / \partial \theta_{i} & \text { Sensitivity of moment with respect to the parameter } \theta_{i} \\ x_{f_{i}}(t) & \text { Displacement of } i \text { th floor } \\ \left(x_{f_{i}}(t)-x_{f_{i-1}}(t)\right) & \text { Inter storey drift } \\ \ddot{u}_{g}(t) & \text { Ground acceleration } \\ D_{c} & \text { Critical displacement of ground floor } \\ m_{f}, k_{f} \text { and }, c_{f} & \text { Mass, stiffness and damping coefficient at each floor } \\ m_{q} & \text { Mean response of } g(\boldsymbol{x}) \\ g_{0} & \text { Simulation size in Monte Carlo simulation } \\ N_{S} & \text { Probability measure } \\ P_{\boldsymbol{\theta}} & \end{array}$




$\begin{array}{ll}N & \text { Number of variables } \\ \mathbf{c} & \text { Reference point } \\ n & \text { Number of sample points }\end{array}$

\section{References}

Alis OF, Rabitz H 2001 Efficient implementation of high dimensional model representations. J. Mathematical Chem. 29(2): 127-142

Au S K 2005 Reliability-based design sensitivity by efficient simulation. Computers and Structures 83(14): 1048-1061

Chowdhury R, Rao B N, Prasad A M 2009 High Dimensional Model Representation for Structural Reliability Analysis. Communications in Numerical Methods in Engineering 25: 301-337

Gavin H P, Yau S C 2008 High-order limit state functions in the response surface method for structural reliability analysis. Structural Safety 30(2): 162-179

Glasserman P 1991 Gradient estimation via perturbation analysis (Boston, MA: Kluwer Academic Publishers)

Haug E J, Choi K K, Komkov V 1986 Design sensitivity analysis of structural systems (New York: Academic Press)

Ho Y C, Cao X R 1991 Discrete event dynamic systems and perturbation analysis (Boston, MA: Kluwer Academic Publishers)

Hohenbichler M, Gollwitzer S, Kruse W, Rackwitz R 1987 New light on first- and second-order reliability methods. Structural Safety 4: 267-284

Lancaster P, Salkauskas K 1986 Curve and surface fitting: An introduction (London: Academic Press)

L'Ecuyer P, Perron G 1994 On the convergence rates of IPA and FDC derivative estimators. Operations Research 42(4): 643-656

Li G, Rosenthal C, Rabitz H 2001 a High dimensional model representations. J. Phys. Chem. A 105(33): 7765-7777

Li G, Wang S W, Rosenthal C, Rabitz H 2001b High dimensional model representations generated from low dimensional data samples-I. mp-Cut-HDMR. J. Mathematical Chem. 30(1): 1-30

Liu P L, Der Kiureghian A 1991 Finite element reliability of geometrically nonlinear uncertain structures. J. Eng. Mech. ASCE 117(8): 1806-1825

Melchers R E, Ahammed M 2004 A fast approximate method for parameter sensitivity estimation in Monte Carlo structural reliability. Computers and Structures 82(1): 55-61

Rabitz H, Alis O F 1999 General foundations of high dimensional model representations. J. Mathematical Chem. 25(2-3): 197-233

Rabitz H, Alis O F, Shorter J, Shim K 1999 Efficient input-output model representations. Computer Phys. Commun. 117(1-2): 11-20

Rubinstein R Y, Shapiro A 1993 Discrete event systems — sensitivity analysis and stochastic optimization by the score function method (New York: John Wiley \& Sons)

Sobol I M 2003 Theorems and examples on high dimensional model representations. Reliability Engineering and System Safety 79(2): 187-193

Wang S W, Levy II H, Li G, Rabitz H 1999 Fully equivalent operational models for atmospheric chemical kinetics within global chemistry-transport models. J. Geophys. Res. 104(D23): 30417-30426

Wen Y K 1976 Method for random vibration of hysteretic systems. J. Eng. Mech. Division, ASCE 102(EM2): 249-263 